\title{
Essential Oils of Herbs and Spices: Their Antimicrobial Activity and Application in Preservation of Food
}

\author{
Silviya R. Macwan ${ }^{1 *}$, Bhumika K. Dabhi ${ }^{2}$, K.D. Aparnathi ${ }^{3}$ and J.B. Prajapati ${ }^{4}$ \\ Dairy Chemistry Department, SMC College of Dairy Science, Anand Agricultural University, \\ Anand- 388001, Gujarat, India \\ *Corresponding author
}

\begin{abstract}
A B S T R A C T
Keywords

Food preservation, antimicrobial compound, essential oil.

Article Info

Accepted:

20 April 2016

Available Online:

10 May 2016

Many food products are perishable by nature and require protection from spoilage during their preparation, storage and distribution. The food products are now often sold in areas of the world far distant from their production sites, and hence need for extended shelf-life products has increased. Several food preservation systems including addition of antimicrobial compounds can be used to enhance shelf-life and reduce the risk of food poisoning. The most common classical preservative agents are the weak organic acids and sodium chloride. However, negative perception against artificial synthetic chemicals has shifted effort towards the development of alternatives that consumers perceive as 'naturals'. Spices and herbs can enhance shelf-life because of their antimicrobial nature, which are primarily added to change or improve taste. Their components are known to contribute to the self-defense of plants against infectious organisms. Therefore, use of essential oils (EOs) of spices and herbs present a better choice than synthetic chemical additives, especially for "organic" food production, which has become popular and is widely accepted by consumers.
\end{abstract}

\section{Introduction}

In the recent years, food safety issues have become one of the main public health concerns. In 2005, WHO reported 1.8 million of death caused by diarrheal diseases, mostly associated with contaminated food and drinking water. Before 1970's Salmonella spp., Shigella spp., Clostridium botulinum, Staphylococcus aureus, Bacillus cereus were recognized as the major causes of gastrointestinal disease, and during the 1980's and 1990's Campylobacter spp., Yersinia enterocolitica, Listeria monocytogenes, Escherichia coli
O157:H7, Vibrio cholerae non O1, Vibrio vulnificus, Norovirus, Cryptosporidium parvum, Cyclospora cayetanensis, Enterobacter sakazakii and prions were added on the list of food pathogens, but it is alarming that in about $50 \%$ of cases causative agents still remain unknown. Salmonella spp., Campylobacter spp., enterohaemorrhagic $E$. coli, including serotype O157:H7, present microbial pathogens of current concern in food, especially in meat, which presents valuable source of proteins, fat, $\mathrm{Fe}$ ion and 
$\mathrm{B}_{12}$ vitamin, and has the main role in human diet, while Listeria monocytogenes can be found in ready-to-eat meat and poultry products. According to farm-to-fork approach in food production, monitoring of foodborne illnesses and pathogens as well as structured approaches to food safety, such as HACCP principles, have been implemented in the food chain. Despite efforts and improvements in slaughter hygiene and food production techniques in food industry, foodborne pathogens found in meat still cause a number of foodborne illness outbreaks yearly all over the world (Marija et al., 2013).

Bacterial evolutionary responses to the antibiotics resulted in the development of bacterial strains resistant to antibiotics (Patil and Kamble, 2011). Considerable postharvest losses of fruit and vegetables are brought about by decay caused by fungal plant pathogens. Fruit, due to their low $\mathrm{pH}$, higher moisture content and nutrient composition are very susceptible to attack by pathogenic fungi, which in addition to causing rots may also make them unfit for consumption by producing mycotoxins (Tripathi and Dubey, 2004). The World Health Organization has recently called for a worldwide reduction in the consumption of salt in order to reduce the incidence of cardio-vascular disease. If the level of salt in processed foods is reduced, it is possible that other additives will be needed to maintain the safety of foods (Burt, 2004).

The recent negative consumer perception against artificial synthetic chemicals, however, has shifted this research effort towards the development of alternatives that consumers perceive as 'naturals'. Therefore a trend about the use of natural additives in foods has been revealed for quite some time as a result of consumer demand and recently the search for natural antioxidants has been widely encouraged (Chorianopoulos et al., 2008).

One such possibility is the use of essential oils (EOs), which present a better choice than some synthetic chemical additives, especially for "organic" and "natural" food production, which has become popular mostly in the Western society, and is widely accepted by consumers (Marija et al., 2013).

The term 'essential oil' is thought to derive from the name coined in the 16th century by the Swiss reformer of medicine, Paracelsus von Hohenheim; he named the effective component of a drug Quinta essential. An estimated 3000 EOs are known, of which about 300 are commercially importantdestined chiefly for the flavours and fragrances market (Burt, 2004).

EOs are volatile, natual, aromatic oily liquids that can be obtained from several parts of the plants especially the aerial ones as leaves and flowers. They are derived from complex metabolic pathways in order to protect the plant organism from diverse pathogenic microorganisms, to repel insects that act as plague vectors, to reduce the appetite of some herbivorous by conferring unpleased taste do the plant. In fact, the large bioactivity of EOs has being confirmed by several studies, including antibacterial, antiviral, anti-inflammatory, antifungal, antimutagenic, anticarcinogenic, and antioxidant as well as other miscellaneous activities (São Pedro et al., 2013).

The use of (EO) dates back to the earliest civilizations: first in the East and the Middle East and later in North Africa and Europe. The Hydrosols (aromatic) were used in India over than 7000 years. Between 3000 and 2000 B.C., the Egyptians made used extensively aromatic plants and other plants to treat the sick. The Persians seem to be the 
first ones who used the hydrodistillation in 1000 B.C (Romane et al., 2012).

\section{Antimicrobial Component}

Essential oils can be liquid at room temperature though a few of them are solid or resinous, and showing different colors ranging from pale yellow to emerald green and from blue to dark brownish red. They are synthesized by all plant organs, i.e., buds, flowers, leaves, stems, twigs, seeds, fruits, roots, wood or bark, and are stored in secretory cells, cavities, canals, epidermiccells or glandular trichomes (Bassolé and Juliani, 2012).

The composition of the EO can vary among different parts of the same plant. Chemically, EO are variable mixtures of principally terpenoids, mainly monoterpenes (C10) and sesquiterpenes (C15), although diterpenes (C20) may also be present, and a variety of low molecular weight aliphatic hydrocarbons, acids, alcohols, aldehydes, acyclic esters or lactones and exceptionally $\mathrm{N}$ - and S-containing compounds, coumarins and homologues of phenylpropanoids (Benchaar et al., 2008). They can be obtained by expression, fermentation, effleurage or extraction but the method of steam distillation is most commonly used for commercial production of EOs (Burt, 2004).

Terpenes are hydrocarbons made up of several isoprene units and terpenes containing oxygen are called terpenoids. Examples of terpenes present in EOs are pcymene, terpinene, limonene, sabinene, and pinene. Terpenoids can be subdivided into alcohols, esters, aldehydes, ketones, ethers, and phenols. Geraniol, menthol, linalool, citronellol, carvone, thymol, carvacrol, geranyl acetate, eugenyl acetate, geranial, neral and 1,8-cineole are the well-known terpenoids found in EOs. Cinnamaldehyde, cinnamyl alcohol, chavicol, eugenol, estragole, methyl eugenols and methyl cinnamate are phenylpropanoids (Jayasena and Jo, 2013).

It has been found that oxygenated monoterpenes, exhibit strong antimicrobial activity, especially pronounced on whole cells, while hydrocarbon derivatives possess lower antimicrobial properties, as their low water solubility limits their diffusion through the medium. Hydrocarbons tend to be relatively bound capacity and water solubility. Ketones, aldehydes and alcohols are active, but with differing specificity and levels of activity, which is related to the present functional group, but also associated with hydrogen-bounding parameters in all cases (Sokovic et al., 2010). Essential oils or their active compounds containing hydroxyl group (-OH) are highly antimicrobial. The presence of aromatic nucleus with a polar functional group determines the inhibitory properties of the essential oils. A hydroxyl group is much more effective compared to a carbonyl group. The hydroxyl group can easily bind the active site of enzymes and alter their metabolism. Besides the presence of the hydroxyl group in the phenolic structure, its position in the phenolic ring strongly influences the microbial effectiveness of components. The alkyl substitution into phenolic compound increases the antimicrobial activity. The type of alkyl substituent in a nonphenolic ring structure affects the antimicrobial activity. An alkenyl substituent $(-\mathrm{CH}=\mathrm{CH}-)$ as in limonene resulted in increased antimicrobial activity compared to an alkyl substituent (-C $=\mathrm{C}-$ ) as in p-cymene (Ceylan and Fung, 2004).

\section{Mechanism of Action}

The antimicrobial activity of EOs, similar to all natural extracts, is dependent on their chemical composition and the amount of the single components. These molecules can be 
naturally present in their active form in the plant or can be activated by specific enzymes when the vegetal organism is subjected to particular biotic or abiotic stress. Their antimicrobial activity is not attributable to a unique mechanism but is instead a cascade of reactions involving the entire bacterial cell; together, these properties are referred to as the "essential oils versatility". The mechanisms of action of the EOs include the degradation of the cell wall, damaging the cytoplasmic membrane, cytoplasm coagulation, damaging the membrane proteins, increased permeability leading to leakage of the cell contents, reducing the proton motive force, reducing the intracellular ATP pool via decreased ATP synthesis and augmented hydrolysis that is separate from the increased membrane permeability and reducing the membrane potential via increased membrane permeability (Nazzaro et al., 2013). An important characteristic of EOs and their components is their hydrophobicity, which enables them to partition in the lipids of the bacterial cell membrane and mitochondria, disturbing the structures and rendering them more permeable. Leakage of ions and other cell contents can then occur (Burt, 2004).

The cell wall of Gram-negative bacteria is more resistant to the activity of EOs and their components. The Gram-negative cell wall does not allow for the entrance of hydrophobic molecules as readily as Grampositive bacteria; thus, EOs are less able to affect the cell growth of the Gram-negative bacteria (Nazzaro et al., 2013).

It is apparent that the generally greater resistance of Gram-negative bacteria to essential oils is likely to be due in part to the greater complexity of the double membranecontaining cell envelope of these organisms in contrast with the single membrane glycoprotein/ techoic acid, or membrane- glycoprotein/ $\beta$-glucan-based structures of Gram positive bacteria and yeast, respectively. Resistance also seems to be related to the rate and extent of antimicrobial dissolution or ability to partition in the lipid phase of the membrane. It is more likely that the effects of differences in hydrophobicity between these two bacterial groups, with Gram-negative cells having more hydrophobic surfaces can be offset by the presence of porin proteins in the outer membrane of Gram -negative cells. These can create channels large enough to allow restricted passage of small molecular mass compounds, like the substituted phenolics in essential oils, allowing their access to the periplasmic space, the glycoprotein layer and the cytoplasmic membrane (Holley and Patel, 2005).

\section{Antibacterial Activity}

The inhibitory effect of thyme, mint, bay leaves and their alcohol extracts on the growth of $S$. typhimurium, $V$. parahaemolyticus and $S$. aureus in a medium was evaluated by Ceylan and Fung, (2004). S. typhimurium was found to be the least sensitive to these spices. Thyme was the inhibitoriest against the bacteria tested. Antimicrobial effect of the essential oils of sage, rosemary, cumin, caraway, clove, and thyme against P. fluorescens, S. marcescens, E. coli, Surcinaspp., Micrococcus spp., S. aureus, B. subtilis. Mycobacterium phlei, and $S$. cerevisiae was evaluated. Clove and thyme essential oils and their active compounds were highly inhibitory against the cultures tested while others exhibited moderate or no antimicrobial activity. The essential oil of bay leaf, pimento berry, pimento leaf and cloves having eugenol as a major component exhibited the minimum inhibitory concentration from 10 to $100 \mathrm{pg}$ against Gram-positive, Gram-negative, and yeast and fungi.. 
Inhibitory action of thyme (Thymus vulgaris), mint (Mentha piperita) and laurel (Laurusnobilis) ground leaves and their extracts on S. aureus, S. typhimurium and $V$. parahaemolyticuswas studied by Souza and co-workers (2005). Thyme was most prominent antibacterial product being active up to concentration $0.5 \%(\mathrm{w} / \mathrm{v})$ for ground and 5000ppm (v/v) for extracts. Testing of antilisterial effect of 18 spices observed significant inhibitory effect of rosemary (Rosmarinus officinalis) and clove (Eugenia cariophylata) on L. monocytogenes. Spices mixtures were able to inhibit the growth of various meat spoiling microorganisms (Bacillus subtillis, Enterococcus spp., Staphylococcus spp., E. coli $\mathrm{K} 12$ and Pseudomonas fluorescens) providing stabilizing effect on colour and smell of fresh portioned pork meat.

The growth and survival of E.coli $\mathrm{O} 157$ and Salmonella enteric serovar enteridis in mayonnaise in presence of garlic (Allium сера), ginger, mustard and ground clove was studied. Garlic and clove which showed bacteriostatic and bactericidal effect, respectively, towards $S$. enteric and E. coli O157, and E. coli was more sensitive (Souza et al., 2005).

In 2007, 46 spice and herb extracts tested by Shan and co-workers, amongst them twelve exhibited high antibacterial activities against the five foodborne bacteria. Methanolic and ethanolic extracts of $P$. granatum were effective against $B$. cereus, E. coli, and $S$. aureus. The extracts and essential oils of $E$. caryophylata, $O$. vulgare, and $C$. burmannii B. had significant inhibitory properties against $S$. aureus, $E$. coli, and $L$. monocytogenes. The ethanolic extracts of $S$. officinalis had strong antimicrobial activity against B. cereus, E. coli, and S. aureus. The extracts of $P$. cuspidatum strongly inhibited the growth of $B$. cereus, $S$. aureus, and $E$. coli. C. auriculata exhibited significant activity against $E$. coli and $S$. aureus.

The antibacterial properties of 14 EOs (clove, oregano, rosemary, pepper, nutmeg, liquorice, turmeric, aniseed, cassia bark, fennel, prickly ash, round cardamom, dahurian angelica root and angelica) against four common meat spoilage and pathogenic bacteria (Listeria monocytogenes, Escherichia coli, Pseudomonas fluorescens and Lactobacillus sake) was studied and their results showed that individual extracts of clove, rosemary, cassia bark and liquorice contained strong antibacterial activity (Škrinjar and Nemet, 2009).

The essential oil from the rhizomes of turmeric (Curcuma longa Syn domestica Val.) exhibited good to moderate activity against $B$ subtilis, $S$. aureus and $C$. diphtheria. Oils of bay clove, cinnamon and thyme can act as potent inhibitors of Listeria monocytogenes and Salmonella enteritidis. Clove, cinnamon, bishop's weed, chili, horse raddish, cumin, tamarind, black cumin, pomegranate seeds, nutmeg, garlic, onion, tejpat, cellary, cmbodge have potent antimicrobial activity against Bacillus subtilis, Escherchia coli and Saccharomyces cerevisiae (Maurya et al., 2013). The antimicrobial properties of fifty plant essential oils against 25 genera of bacteria were evaluated. Thyme, cinnamon, bay, clove, almond (bitter), lovage, pimento, marjoram, angelica and nutmeg essential oils displaced the greatest inhibitory properties. Clove extract showed remarkable antibacterial activity against all organisms tested and oregano and cinnamon exhibited wide inhibitory spectrum (Mohamed et al., 2013).

\section{Antimold Activity}

Spoilage and poisoning of foods by fungi is a major problem, especially in developing countries. Penicillium, Aspergillus and 
Fusarium are the most important fungi causing spoilage of African foodstuffs (Nguefack et al., 2004).

Carvone, a monoterpene, isolated from the essential oil of Carumcarvihas been shown to inhibit sprouting of potatoes during storage and it also exhibited fungicidal activity in protecting the potato tubers from rotting without altering taste and quality of the treated commodity, and without exhibiting mammalian toxicity. It has been introduced under the trade name TALENT in The Netherlands (Tripathi and Dubey 2004).

In addition to inhibition of vegetative growth, the other oils also inhibited production of mycotoxins by fungi. Thyme, anise and cinnamon oils were able to inhibit production of aflatoxins, ochratoxins $\mathrm{A}$ and fumonisin in broth at $2 \%$. Anise oil showed fungicidal activity at $500 \mathrm{ppm}$, while caraway and fennel oil at 2000 ppm were required to inhibit all four fungi. The oils of lavender, rosemary and sage gave mycelial inhibition. Thyme, oregano and dictamus oils were fungitoxic and this may have been due to formation of hydrogen bonds between the hydroxyl group of oil phenolics and active sites of target enzymes (Holley and Patel, 2005).

Effectiveness of nine essential oils to control the growth of mycotoxins producing moulds was studied and noted that clove, cinnamon and oregano were able to prevent the growth of Aspergillus parasiticus and Fusarium moniliforme, while clove (ground and essential oil) markedly reduced the aflatoxin synthesis in infected grains (Souza et al., 2005).

Lemongrass oil revealed the greatest potential of antimicrobial activity against all test organisms (10 molds, 2 yeasts and 5 bacteria). This oil was followed by basil, thyme and origanum oils. The essential oil of Ocimum basilicum at a concentration of 1.5 $\mathrm{ml} / \mathrm{l}$ completely suppressed the mycelia growth of 22 species of fungi including the mycotoxin producing strains of $A$. flavus and A. parasiticus (Helal et al., 2006).

The effect of the essential oils of lemon (Citrus lemon L.), mandarin (Citrus reticulata $L$.), grapefruit (Citrus paradisi L.) and orange (Citrus sinensis L.) on the growth of moulds commonly associated with food spoilage was investigated: Aspergillus niger, Aspergillus flavus, Penicillium chrysogenum and Penicillium verrucosum, using the agar dilution method. The order of oil efficacy against these moulds was grapefruit> lemon>orange> mandarin. An inhibitory effect of various concentrations of mint (Mentha piperita L.) and caraway (Carvum carvi L.) on the growth of some toxigenic Aspergillus species and aflatoxin B1 production was examined. Mint showed stronger inhibitory effect than caraway (Škrinjar and Nemet, 2009).

The essential oil from $C$. longa has been studied for its biological activities, such as its antifungal activity. The essential oil from C. longa at 1000 ppm (0.1\%) inhibited Fusarium moniliforme mycelial growth. The same result occurred at 2000ppm (0.2\%) for Aspergillus niger and Fusarium oxysporum. Essential oils from Thymus vulgaris, Lavandula, and Mentha piperita caused the degeneration of hyphae and cytoplasmatic emptying in Colletotrichum lindemuthianum and Pythium ultimum var. ultimumwas demonstrated. A marked reduction in $A$. niger conidiophore formation after Matricaria chamomilla L. essential oil treatment was observed. The antifungal activity of essential oils from aromatic plants on A. fumigatus and Trichophyton rubrum was associated with the damage to 
the cell wall and cytoplasmic contents. Furthermore, the lipophilic properties of essential oils allow them to penetrate the plasma membrane, causing polysaccharide accumulation under drought stress conditions and leading to plasmalemma breakage in fungal cells. (Ferreira et al., 2013)

\section{Anti-Yeast Activity of Essential Oils}

Anti-yeast effect of EOs was mainly tested by disc diffusion methods. Oregano and thyme EOs are apparently among the best inhibitors Other EOs, such as juniper, lemon, marjoram, clary sage, basil, ginger or lemon balm, containing non-phenolic main compounds have been also found to show high toxicity against yeasts. The susceptibility of yeasts against 12 EOs in decreasing order was: $S$. pombe> $S$. cerevisiae >Yarrowia lipolytica> Rhodotorula glutinis with MIC values from $0.03 \mathrm{mg} / \mathrm{ml}$ up to $0.54 \mathrm{mg} / \mathrm{ml}$ (Krisch et al., 2011).

\section{Factors Affecting Antimicrobial Activity}

In the antimicrobial action of essential oil components, the lipophilic character of their hydrocarbon skeleton and the hydrophilic character of their functional groups are of the main importance. The activity rank of essential oil components is as follows: phenols $>$ aldehydes $>$ ketones $>$ alcohols $>$ ethers > hydrocarbons (Kalemba and Kunicka, 2003).

In addition, both intrinsic and extrinsic conditions can be responsible to the susceptibility or resistant nature of the pathogen. Besides, fatty contents may also serve as barrier to the effectiveness of the pathogen while incorporating plant essential oils (PEO) in foods due to having a higher population of pathogen in hydrophilic region. Hence, it is assumed that fatty composition may directly have an adversary effect on the efficacy of PEOs against the tested pathogens. In addition, interaction of terpenoid phenolics of PEOs with enzymatic substances might be a reason to limit the antimicrobial efficacy of the PEOs in food models (Bajpai et al., 2012).

There are a host of other factors, variability in composition or content of active agents that result from agronomic history, varietal differences, and maturity of the plant material studied; physical and chemical characteristics of the antimicrobial itself(hydrophobicity, volatility, compatibility in the test system); presence of protein , starch or lipid that may complex with and neutralize antimicrobial activity or partition the agent away from its target; and the inoculum size, genus of microorganism, species and even strain susceptibility as well as previous culture history (Holley and Patel, 2005).

The efficiency of an antimicrobial compound depends on the type, genus, species, and strain of the target microorganism, besides the environmental factors such as $\mathrm{pH}$, water activity, temperature, atmospheric composition and initial microbial load of the food substrate. The antimicrobial nature of phytochemical is determined by its chemical properties, such as $\mathrm{pKa}$ value, hydrophobicity/ lipophilicity ratios, solubility, and volatility. The $\mathrm{pH}$ and polarity are the most prominent factors influencing the effectiveness of a food antimicrobial (Negi, 2012).

Hydrophobic properties of some antimicrobial substances can make their dissolution difficult in water limiting their use in foods. The concentration thresholds required for inhibition or inactivation of microorganisms will depend on the specific targets of the antimicrobial substance, including cell wall, cell membrane, 
metabolic enzymes, protein synthesis, and genetic systems (Negi, 2012).

\section{Methods used to Study AMA}

Tests of antimicrobial activity can be classified as diffusion (Agar disk diffusion assay \& Agar well diffusion), dilution (Broth microdilution \& Agar dilution assay (Ncube et al., 2008) or bio autographic methods. Also killing kinetics \& interaction testing (Paparella et al., 2013) are also used. However, since the outcome of a test can be affected by factors such as the method used to extract the EO from plant material, the volume of inoculum, growth phase, culture medium used, $\mathrm{pH}$ of the media and incubation time and temperature, comparison of published data is complicated (Burt, 2004).

Evaluation of the performance of a susceptibility test should include criteria such as ease of use, reproducibility, i.e. the ability to yield the same result on repeat testing, test sensitivity and specificity. Although current standard methods, approved by various bodies like the National Committee for Clinical Laboratory Science (NCCLS) [now known as Institute of Clinical Laboratory Standards (ICLS)], British Society for Antimicrobial Chemotherapy (BSAC) and the European Committee for Antimicrobial susceptibility testing (EUCAST), exist for guidelines of antimicrobial susceptibility testing of conventional drugs, these might not be exactly applicable to plant extracts and modifications have to be made (Ncube et al., 2008).

\section{Application}

\section{Component in Packaging Material}

Active Packaging (AP) is an innovative food packaging concept that combines advances in food technology, food safety, and packaging and material sciences in an effort to better meet consumer demands for freshlike, safe products. The use of antimicrobial packaging techniques as a promising type of active packaging (Matan et al., 2006). In antimicrobial packaging, antimicrobial agents can be incorporated into the packaging material, coated on the surface of packaging film or a sachet containing antimicrobial compound can be added into the package (Emiroğlu et al., 2010). Antimicrobial packaging that interacts with the head space consists of a packaging system using a sachet, which is a pad containing volatile antimicrobial agents, enclosed in the interior of the packaging or attached to it. The migration of the active compound may be achieved by direct contact between food and the packaging material or through gas phase diffusion from the inner packaging layer to the food surface (Ramos et al., 2013).

Addition of EOs to a wax coating in order to develop an antimicrobial active packaging was studied and ability to preserve strawberries from microorganism contamination by the release of AMs from the coating was assessed. In other study, carvacrol was added to chitosan-based films for active packaging and its antimicrobial efficiency against food spoilage microorganisms was demonstrated by using a headspace chromatographic technique. Cinnamon-based active material was used to increase more than three times the shelf-life of a complex bakery product with minimal changes in the packaging and no additional manipulation steps (Ramos et al., 2013).

\section{As a Component in Hurdle Technology}

The hurdle effect is defined as the contribution of several factors to produce stable and safe products. Heating, chilling, freezing, freeze-drying, curing, salting, sugar-addition, acidification, fermentation, 
smoking and oxygen removal are some processes used in food processing. In general these parameters or hurdles can be classified as high temperature, low temperature, water activity (aw) level, $\mathrm{pH}$, redox potential, preservative and competitive flora (Ceylan and Fung, 2004).

In addition, the necessary concentration of essential oils and their components to achieve an antimicrobial effect in foods is much higher than under in vitro conditions. The presence of salt, proteins, fat, carbohydrates, and a low aw-value cause a decrease of the antimicrobial properties of essential oils (Klein et al., 2013).

Essential oils have been used in combination with other antibacterial agents and with a variety of treatments such as mild heat, hydrostatic pressure, sodium citrate and monolaurin. Recently, synergistic effects of essential oils and nisin on Bacillus cereus and Listeria monocytogenes was reported, and the addition of lysozyme as a third preservative factor enhances the synergistic effect between carvone and nisin (Yamazaki et al., 2004).

The microbial stability of apple juice supplemented with extracts of lemon grass, clove, rosemary, basil and sage during storage at refrigeration $\left(4^{\circ} \mathrm{C}\right)$ for 4 weeks was investigated. Heating the mango juice at $55^{\circ} \mathrm{C}$ for $15 \mathrm{~min}$ and supplementing with $4 \%$ nutmeg and $4 \%$ ginger markedly inhibited microbial growth and produced a product with acceptable taste (Eissa et al., 2008).

\section{Prevention of Toxin Production}

Mycotoxins are secondary metabolites produced by specific filamentous fungi that contaminate agricultural commodities. They are toxic to humans and animals, cause significant reductions in crop yield and cause economic losses. Food contaminated with mycotoxins, particularly with aflatoxins, can cause sometimes fatal acute illness, and are associated with increased cancer risk (Yazdani et al., 2013, Rasooli et al., 2008).

The high health risk caused by aflatoxins leads to strict concentration limits in different countries. The US Food and Drug Administration (USFDA) established $20 \mathrm{ppb}$ as the minimum acceptable level of aflatoxin in all foods other than milk. The European Union has banned the import of peanut with $>2$ ppb of AFB 1 content and with $>4$ ppb of total aflatoxins in nuts prepared for human consumption. To export tree nuts to the European market, aflatoxin levels should be $>3$ ppb (Sindhu et al., 2011).

Plant extracts or essential oils can provide potential alternatives to the currently used toxic fungicides to control post-harvest fungal deterioration. The biosynthesis of aflatoxin B1 can be inhibited by extracts of certain plants that are toxic to fungi and may be useful in controlling the fungal growth and mycotoxin production. Extracts of plants such as garlic and onion effectively retard growth and aflatoxin production. Effect of turmeric leaf oil on the fungal growth and aflatoxin production was determined (Sindhu et al., 2011).

Essential oils from 16 aromatic plants, i.e., safflower, marigold, coriander, pomelo, mangosteen, Kaempferia parviflora, ginger, pepper, Boraphet, aloe, lavender, rosemary, cinnamon, eucalyptus, thyme, and white wood were tested for their inhibitory effect on A. flavusIMI 242684 on PDA by agar diffusion test. $100 \mathrm{ppm}$ of five oils, namely geraniol, nerol and citronellol (aliphatic oils), cinnamaldehyde (aromatic aldehyde) and thymol (phenolic ketone), each completely suppressed growth of $A$. flavusand consequently prevented aflatoxin synthesis in liquid medium (Thanaboripat, 2011). 
Crude extract, chloroform and water fractions prepared from $P$. betledried leave did not show any inhibitory effect against $A$. flavusat concentration $1-5 \mathrm{mg} / \mathrm{ml}$ in disk diffusion test. However, a significant reduction in aflatoxin B1 production was observed for $P$. betlechloroform fraction. This fraction at $500 \mu \mathrm{g} / \mathrm{ml}$ caused a reduction (91\%) in mycelial growth and completely inhibited toxin biosynthesis by A. flavusin present experiment (Yazdani et al., 2013).

\section{Edible Films}

Edible films are thin films prepared from edible material that act as a barrier to external elements (factors such as moisture, oils, gases and vapors) and thus protect the product, extend its shelf life and improve its quality. Edible films can control moisture, oxygen, carbon dioxide, flavor and aroma transfer between food components or the atmosphere surrounding the food (Du et al., 2011). In recent years, packaging research has focused more on biodegradable films, including films made from plant and animal edible protein sources such as corn zein, wheat gluten, soy and peanut protein, cottonseed, albumin, gelatin, collagen, casein and whey proteins. Milk protein based edible films have good mechanical strength and are excellent oxygen, lipid, and aroma barriers; however, due to their hydrophilic nature, they have poor moisture barrier properties. This property was improved by the incorporation of hydrophobic materials such as lipids (Seydim and Sarikus, 2006).

Addition of EOs to edible films and coatings prevents the growth of pathogenic and spoilage bacteria. It further enhances sensory properties in foods including meat and meat products. Wrapping pieces of chicken breast with apple films containing $0.5 \%$ carvacrol or cinnamaldehyde- active ingredients of oregano and cinnamon oils, respectively, reduced the growth of $E$. coli O157:H7, Salmonella enterica, and $L$. monocytogenes after storage at $4{ }^{\circ} \mathrm{C}$ for $72 \mathrm{~h}$ and that the bactericidal activity was greater with carvacrol than with cinnamaldehyde. Additionally, tomato-based film-forming solution with carvacrolwas effective against E. coli O157:H7. Similarly, edible films such as apple and tomato films containing carvacrol or cinnamaldehyde can be used to protect raw chicken pieces against bacterial contamination; this treatment did not change the sensory qualities of the baked wrapped chicken and contributed to the nutritional and health benefits of the wrapped chicken pieces. Carvacrol is more compatible with tomato wraps, while a similar concentration of cinnamaldehyde is more effective with apple wraps used on baked chicken pieces (Jayasena and Jo, 2013).

\section{Prevention and Reduction of Biofilm Formation}

Biofilms comprise sessile microbial communities surrounded by a matrix of extracellular polymeric substances (EPS), such as proteins, nucleic acids and polysaccharides. This bacterial phenotype is an example of physiological adaptation, which is more difficult to eliminate, and is one of the most important sources of recalcitrant infections.

Biofilm formation is an extremely common phenomenon and most materials in contact with a natural fluid may rapidly become colonized by bacteria. In the majority of natural environments, monospecies biofilms are relatively rare. Conversely, microorganisms are associated with surfaces in complex multispecies communities. In the food industry of fermented products (e.g. traditional sausages), mixed bacterial biofilms may contain strains beneficial to 
the quality of the food product (e.g. lactobacilli and staphylococci). However, biofilms of both spoilage and pathogenic microflora are related to problems of food contamination, leading to lowered shelf-life of products and transmission of diseases. The risk becomes even more serious since bacteria in biofilms have been shown to express increased resistance to disinfectants compared with their planktonic counterparts (Chorianopoulos et al., 2008). Nowadays, it is priority for food-processing industries to apply well cleaning and sanitation programmes to minimize the risk of biofilm contamination. However, this is not always easy to accomplish because used disinfectants, in order to be effective, should be able to penetrate the extracellular polymeric substances matrix surrounding the biofilms and kill the cells (Chorianopoulos et al., 2008).

Essential oils of lavender, tea tree, and lemon balm have been shown to have antibiofilm activity using Staphylococcus aureus and Escherichia coli as test organism. Essential oils from two Bowelliasp. (Frankincense oil) have the ability to inhibit biofilm formed by two species of Staphylococcus and Candida albicans. Peppermint oil has also been shown to inhibit biofilm formed by $C$. albicans (Mukherji and Prabhune, 2014).

Methanolic extracts of Cuminum cyminum, which contain methyl eugenol, an EO with an aromatic ring, inhibited swimming and swarming motility, Qurom sensing (QS), Exopolysacharide production and biofilm formation by $P$. aeruginosa, Proteus mirabilis and Serratia marcescens. EO from Cinnamomum cassia and their components, cinnamaldehyde and eugenol, affected the biofilm formation by Escherichia coli, interfering with their swimming motility, potential of adhesion and structure. Moreover, cinnamaldehyde, inhibited QS of
E. coli and Vibrio harveyi, affecting acyl homoserine lactones and autoinducer-2 that mediate QS. Plant EO, such as clove, cinnamon, peppermint and lavender, also exhibited QS inhibition (QSI) (Borges et al., 2013).

Anti-adhesive properties of some polyphenolic products against the ability of Streptococcus mutansto adsorb to salivacoated hydroxyapatite and glass, as adhesion surfaces, were observed. In another study, the anti-biofouling effects of polyphenols and the relationship between the inhibition of enzymes produced by $S$. mutansand their biofilm formation ability was investigated and it was found that enzyme inhibition can lead to a decreased biofilm formation. Biofilm formation of various $S$. aureus strains, was inhibited by extracts of RubusulmifoliusSchott, Rosaceae, which are rich in the polyphenol ellagic acid and glycosylated derivatives. Ellagic acid alone was also shown to possess antibiofilm properties (Borges et al., 2013).

\section{Synergism}

Antimicrobial activity of essential oils is conditioned by the activity of their components (Kalemba and Kunicka, 2003).

The interaction between EO compounds can produce four possible types of effects: indifferent, additive, antagonistic, or synergistic effects. An additive effect is observed when the combined effect is equal to the sum of the individual effects. Antagonism is observed when the effect of one or both compounds is less when they are applied together than when individually applied. Synergism is observed when the effect of the combined substances is greater than the sum of the individual effects while the absence of interaction is defined as indifference. 
Literature survey has demonstrated that an oil as a whole showed better antibacterial efficacy than only a combination of major volatiles of the oil. Hence, it might be said that the minor elements of the oil have crucial roles to increase the biological effectiveness of the oil, resulting in the synergism (Bajpai et al., 2012).

There are some generally accepted mechanisms of antimicrobial interaction that produce synergism: sequential inhibition of a common biochemical pathway, inhibition of protective enzymes, combinations of cell wall active agents, and use of cell wall active agents to enhance the uptake of other antimicrobials (Goñi et al., 2009).

Synergism between carvacrol and p-cymene, a very weak antimicrobial, might facilitate carvacrol's transportation into the cell by better swelling the B. cereus cell wall. Thymol and carvacrol showed synergistic and antagonistic effects, in different combinations of cilantro, coriander, dill and eucalyptus EOs and mixtures of cinnamaldehyde and eugenol, against Staphylococcus sp., Micrococcus sp., Bacillus sp. and Enterobacter sp. Vacuum packing in combination with oregano EOs showed a synergistic effect against $L$. monocytogenes with 2-3 $\log 10$ reduction. Similar results have been recorded when clove and coriander EOs have been used against Aeromonas hydrophila on vacuum packed pork. Application of oregano EO has a synergistic effect in modified-atmosphere packaging (MAP) including $40 \% \mathrm{CO}_{2}, 30 \%$ $\mathrm{N}_{2}$ and $30 \% \mathrm{O}_{2}$ (Tajkarimi, et al., 2010).

Application of nisin with carvacrol or thymol has been positively effective against Bacillus cereus with temperatures increasing from 8 to $30^{\circ} \mathrm{C}$. Application of nisin with rosemary extract enhanced the bacteriostatic and bactericidal activity of the nisin.
Oregano EOs, in combination with MAP, have effectively increased the shelf life of fresh chicken. Combinations of EOs of oregano and thyme, oregano with marjoram and thyme with sage had the most effects against Bacillus cereus, Pseudomonas aeruginosa, Escherichia coli O157:H7 and $L$. monocytogenes (Tajkarimi, et al., 2010).

Synergistic effects of eugenol/carvacrol and eugenol/thymol might be due to the fact that carvacrol and thymol disintegrated the outer membrane of $E$. coli, making it easier for eugenol to enter the cytoplasm and combine with proteins. It was also observed the synergistic effect of eugenol/ cinnamaldehyde is probable due to the interaction of these components with different proteins or enzymes. Thymol or carvacrol could increase the permeability of the cytoplasmic membrane, and probably enable cinnamaldehyde to be more easily transported into the cell. Thymol or carvacrol could increase the number, size or duration of existence of the pores created by the binding of cinnamaldehyde to proteins in the cell membrane, so that a synergistic effect is achieved when these two components are used in combination. The synergistic combinations of EOs of oregano/basil against $E$. coli, basil/bergamot against $S$. aureus, oregano/bergamot against B. subtilis and oregano/perilla against $S$. cerevisiae significantly disrupted the integrity of cell membranes when compared with control untreated membranes (Bassolé and Juliani, 2012).

The combination of clove and rosemary essential oils produced an additive effect against the Gram-positive and Gramnegative bacteria, namely $S$. aureus, $S$. epidermidis, Bacillus subtilis, Escherichia coli, Proteus vulgaris and $P$. aeruginosa (Faleiro, 2013). 
The exploitation of the synergetic action resulting from the combination of EO with other biological antimicrobial substances such as bacteriocins has been investigated. The use of oregano or savory EO showed a synergistic activity with a combination of cell-adsorbed bacteriocin of a Lactobacillus curvatus strain when applied to control Listeria monocytogenes in pork meat storage at $4^{\circ} \mathrm{C}$.

The association of the EO components, thymol or cinnamaldehyde with low temperatures $\left(\geq 8^{\circ} \mathrm{C}\right)$ also produces a synergistic effect on the control of the foodborne bacteria B. cereus. (Faleiro, 2013)

Synergism between carvacrol and its biological precursor p-cymene has been noted when acting on $B$. cereus vegetative cells. It appears that p-cymene, a very weak antibacterial, swells bacterial cell membranes to a greater extent than carvacrol does. By this mechanism p-cymene probably enables carvacrol to be more easily transported into the cell so that a synergistic effect is achieved when the two are used together. Synergism between $\mathrm{NaCl}$ and mint oil against $S$. enteritidis and $L$. monocytogenes has been recorded in taramosalata. The combined use of $2-3 \%$ $\mathrm{NaCl}$ and $0.5 \%$ clove powder (containing eugenol and eugenyl acetate) in mackerel muscle extract has been found to totally prevent growth and histamine production by E. aerogenes (Burt, 2004).

Three plants extract of cornifructus, cinnamon and Chinese chive showed strong inhibitory effect on bacteria, on yeasts and on moulds, respectively. Hence, extracts of cornifructus, cinnamon and Chinese chive were mixed at the ratio of 1:1:1 ( v/v/v), and proven to possess the distinctly antimicrobial effect on 15 test microorganisms (Hsieh et al., 2001).

\section{Limitation}

The limitation of the oils' activity can be explained by the low water solubility of the oil and its components which limits their diffusion through the agar medium in the disc-diffusion method (Soković, et al., 2010). As has been shown by some other researchers, the use of antimicrobials can reduce or eliminate specific microorganisms but it may also produce favourable conditions for other microorganisms (Škrinjar and Nemet, 2009). It may be difficult to maintain quality consistency because the composition of an individual EO can vary due to several factors including the time of harvesting, variety, the part of the plant used, and method of extraction. In addition, the antimicrobial potency of EO constituents depends on $\mathrm{pH}$, temperature, and level of microbial contamination (Jayasena and Jo, 2013).

In several reports, high concentrations of EO (components) were needed to reduce growth of L. monocytogenes in cheese and minced meat, chicken, ham, and liver sausage. A similar need for higher concentrations of EOs has been reported for other food products such as fish, dairy products, and vegetables, although a few exceptions exist. The higher concentrations needed often cannot be used in practice because of the aroma connected to these EOs. It is generally believed that the higher fat and protein contents of food compared with growth medium is the major cause of this higher microbial resistance to EOs.

One hypothesis is that the food product provides more nutrients to bacteria, thereby enabling them to repair damaged cells more quickly. Another suggestion is that EO components can dissolve in the fat-lipid phase of the food, which would make these components less available to act on bacteria 
present in the water phase of these foods (Veldhuizen et al., 2007).

Although essential oils possess antibacterial properties and may improve taste and some other characteristics of the meat, they should be used with care, because EOs may cause some side effects. Some essential oils, such as menthol, eugenol and thymol, depending on concentration, may cause irritation of mucous membranes, probably as a result of membrane lysis and surface activity, while cinnamaldehyde, carvacrol, carvone and thymol in vitro appear to have mild to moderate toxic effects at the cellular level (Marija et al., 2013).

\section{Legal Aspects of the Use of Essential Oils}

The European Union (EU) and FDA are constantly producing the most valuable recommendations and laws on the issues of use of essential oils in various industries. A number of essential oils have been registered by European Commission and FDA for their use in food and agriculture industries to control harmful pathogenic microbes. The EU registered essential oils also appear on the 'Everything Added to Food in the US (EAFUS) list, which means that FDA has classified these substances as generally recognized as safe (GRAS) (Bajpai et al., 2011).

In India, according to Food Safety and Standards (Food Products Standards and Food Additives) Regulations, 2011, spices are considered as Class I preservative. Addition of Class I preservatives in any food is not restricted, unless otherwise provided in the regulations.

In conclusion, food contamination is enormous public health problem, but it could be controlled by the use of natural preservatives such as essential oils obtained from spices. The fact that many EOs possess antimicrobial activity has been proved by plenty of investigations in the recent years. The type and optimal concentration of EO depend on the product used and against which species of bacteria or fungi it is to be used. But if EOs are expected to be widely applied as antibacterial and antifungals, the organoleptical impact should be considered as the use of naturally derived preservatives can alter the taste of food or exceed acceptable flavour thresholds. Therefore, research in this area should be focused on the optimization of EO combinations and applications to obtain effective antimicrobial activity at sufficiently low concentrations so as not to adversely influence the organoleptic acceptability of the foods.

\section{References}

Bajpai, V.K., Baek, H.K., Kang, S.C. 2012. Control of Salmonella in foods by using essential oils: A review. Food Res. Int., 45: 722-734.

Bajpai, V.K., Kang, S., Xu, H., Lee, S.G., Baek, K.H., Kang, S.C. 2011. Potential roles of essential oils on controlling plant pathogenic bacteria Xanthomonas species: A review. Plant Pathol. J., 27: 207-224.

Bassolé, I.H.N., Juliani, H.R. 2012. Essential oils in combination and their antimicrobial properties. Mol., 17: 39894006.

Benchaar, C., Calsamiglia, S., Chaves, A.V., Fraser, G.R., Colombatto, D., McAllister, T.A., Beauchemin, K.A. 2008. A review of plant-derived essential oils in ruminant nutrition and production. Animal Feed Sc. Technol., 145: 209-228.

Borges, A., Abreu, A.C., Malheiro, J., Saavedra, M.J., Simões, M. 2013. Biofilm prevention and control by dietary phytochemicals. In "Microbial pathogens and strategies for combating them: science, technology and 
education", (A. Méndez-Vilas Ed.). Formatex Research Center, Zurbaran, 06002 Badajoz, Spain.

Burt, S. 2004. Essential oils: their antibacterial properties and potential applications in foods: A review. Int. J. Food Microbiol., 94: 223-253.

Ceylan, E., Fung, D.Y.C., Sabah, J.R. 2004. Antimicrobial activity and synergistic effect of cinnamon with sodium benzoate or potassium sorbate in controlling Escherichia coli O157:H7 in apple juice. J. Food Sc., 69: FMS102FMS106.

Chorianopoulos, N.G., Giaouris, E.D., Skandamis, P.N., Haroutounian, S.A., Nycha, G.J.E. 2008. Disinfectant test against monoculture and mixed-culture biofilms composed of technological, spoilage and pathogenic bacteria: bactericidal effect of essential oil and hydrosol of Saturejathymbra and comparison with standard acid-base sanitizers. J. Applied Microbiol., 104: 1586-1596.

Du, W.-X., Avena-Bustillos, R.J., Hua, S.S.T., McHugh, T.H. 2011. Antimicrobial volatile essential oils in edible films for food safety. In "Science against microbial pathogens: communicating current research and technological advances" (A. Méndez-Vilas Ed.). FORMATEX, C/ Zurbarán 1, $2^{\circ}$ Oficina 1, 06002 Badajoz, Spain. PP 1124-1134.

Eissa, H.A., Abd-Elfattah, S.M., Abu-Seif, F.A. 2008. Antimicrobial, antibrowning and antimycotoxigenic activities of some essential oil extracts in apple juice. Pol. J. Food Nutr. Sc., 58: 425432.

Emiroğlu, Z.K., Yemi, G.P., Coskun, B.K., Candogan, K. 2010. Antimicrobial activity of soy edible films incorporated with thyme and oregano essential oils on fresh ground beef patties. Meat Sc., 86: 283-288.

Faleiro, M.L. 2013. The mode of antibacterial action of essential oils. In "Microbial pathogens and strategies for combating them: science, technology and education", (A. Méndez-Vilas Ed.). Formatex Research Center, Zurbaran, 06002 Badajoz, Spain. PP 1143-1156.

Ferreira, F.D., Mossini, S.A.G., Ferreira, F.M.D., Arrotéia, C.C., da Costa, C.L., Nakamura, C.V., Machinski, M. 2013. The inhibitory effects of Curcuma longa L. essential oil and curcumin on Aspergillus flavuslink growth and morphology. Scientific World J., doi.org/10.1155/2013/343804.

Goñi, P., López, P., Sánchez, C., Gómez-Lus, R., Becerril, R., Nerín, C. 2009. Antimicrobial activity in the vapour phase of a combination of cinnamon and clove essential oils. Food Chem., 116: 982-989.

Helal, G.A., Sarhan, M.M., Abu Shahla, A.N.K., Abou El-Khair, E.K. 2006. Antimicrobial activity of some essential oils against microorganisms deteriorating fruit juices. Mycobiol., 34: 219-229.

Holley, R.A., Patel, D. 2005. Improvement in shelf-life and safety of perishable foods by plant essential oils and smoke antimicrobials. Food Microbiol., 22: 273-292.

Hsieh, P.C., Mau, J.L., Huang, S.H. 2001. Antimicrobial effect of various combinations of plant extracts. Food Microbiol., 18: 35-43.

Jayasena, D.D., Jo, C. 2013. Essential oils as potential antimicrobial agents in meat and meat products: A review. Tren. Food Sc. Technol., 34: 96-108.

Kalemba, D., Kunicka, A. 2003. Antibacterial and antifungal properties of essential oils. Curr. Med. Chem., 10: 813-829.

Klein, G., Ruben, C., Upmann, M. 2013. Antimicrobial activity of essential oil components against potential food spoilage microorganisms. Curr.. Microbiol., 67: 200-208.

Krisch, J., Tserennadmid, R., Vágvölgy, C. 2011. Essential oils against yeasts and moulds causing food spoilage. In 
"Science against microbial pathogens: communicating current research and technological advances" (A. MéndezVilas Ed.). Formatex Research Center, Zurbaran, 06002 Badajoz, Spain. PP 1135-1142.

Marija, B., Milan, B.Z., Jelena, I., Jelena, D., Jasna, L., Marija, D., Radmila, M. 2013. Use of essential oils in order to prevent foodborne illnesses caused by pathogens in meat. Tehnologija mesa, 54: 14-20.

Matan, N. 2012. Antimicrobial activity of edible film incorporated with essential oils to preserve dried fish (Decapterusmaruadsi). Int. Food Res. J., 19: 1733-1738.

Maurya, S., Kushwaha, A.K., Singh, G. 2013. Biological significance of spicy essential oils. Adv. Nat. Sc., 6: 84-95.

Mohamed, S.H.S., Zaky, W.M., Kassem, J.M., Abbas, H.M., Salem, M.M.E., Said-Al Ahl, H.A.H. 2013. Impact of antimicrobial properties of some essential oils on cheese yoghurt quality. World Appl. Sc. J., 27: 497-507.

Mukherji, R., Prabhune, A. 2014. Novel glycolipids synthesized using plant essential oils and their application in quorum sensing inhibition and as antibiofilm agents. Scientific World J., 1-7.

Nazzaro, F., Fratianni, F., De Martino, L., Coppola, R., De Feo, V. 2013. Effect of Essential Oils on Pathogenic Bacteria. Pharma., 6: 1451-1474.

Ncube, N.S., Afolayan, A.J., Okoh, A.I. 2008. Assessment techniques of antimicrobial properties of natural compounds of plant origin: current methods and future trends. Afr. J. Biotechnol., 7: 17971806.

Negi, P.S., Jayaprakasha, G.K., Jagan Rao Mohan, L., Sakariah, K.K. 1999. Antibacterial activity of turmeric oil: a byproduct from curcumin. J. Agril. Food Chem., 47: 4297-4300.

Nguefack, J., Leth, V., AmvamZollo, P.H., Mathur, S.B. 2004. Evaluation of five essential oils from aromatic plants of
Cameroon for controlling food spoilage and mycotoxin producing fungi. Int. J. Food Microbiol., 94: 329-334.

Paparella, A., Serio, A., López, C.C., Mazzarrino, G. 2013. Plant-based intervention strategies for Listeria monocytogenes control in foods. In "Microbial pathogens and strategies for combating them: science, technology and education", (A. Méndez-Vilas Ed.). Formatex Research Center, Zurbaran, 06002 Badajoz, Spain. PP 1412-1418. PP 1230-1246.

Patil, S.D., Kamble, V.A. 2011. Antibacterial activity of some essential oils against food borne pathogen and food spoilage bacteria. Int. J. Pharma Biosc., 2: B114B150.

Ramos, M., Beltran, A., Valdes, A., Peltzer, M.A., Jimenez, A., Garrigos, M.C., Zaikov, G.E. 2013. Carvacrol and thymol for fresh food packaging. $J$. Bioequiv. Availab., 5: 154-160.

Rasooli, I., Fakoor, M.H., Yadegarinia, D., Gachkar, L., Allameh, A., Rezaei, M.B. $2008 . \quad$ Antimycotoxigenic characteristics of Rosmarinus officinalis and Trachyspermumcopticum L. essential oils. Int. J. Food Microbiol, 122: 135-139.

Romane, A., Harrak, R., Bahri, F. 2012. Use thyme essential oils for the prevention of salmonellosis. In 'Salmonella - A Dangerous Foodborne Pathogen", (Barakat S.M. Mahmoud Ed.). InTech Open Access Company, JanezaTrdine 9, 51000 Rijeka, Croatia. PP 305-332.

São Pedro, A., Espirito Santo, I., Silva, C.V., Detoni, C., Albuquerque, E. 2013. The use of nanotechnology as an approach for essential oil-based formulations with antimicrobial activity. In "Microbial pathogens and strategies for combating them: science, technology and education", (A. Méndez-Vilas Ed.). Formatex Research Center, Zurbaran, 06002 Badajoz, Spain. PP 1364-1374.

Seydim, A.C., Sarikus, G. 2006. Antimicrobial activity of whey protein based edible 
films incorporated with oregano, rosemary and garlic essential oils. Food Res. Int., 39: 639-644.

Shan, B., Cai, Y.-Z., Brooks, J.D., Corke, H. 2007. The in vitro antibacterial activity of dietary spice and medicinal herb extracts. Int. J. Food Microbiol., 117: 112-119.

Sindhu, S., Chempakam, B., Leela, N.K., Bhai, R.S. 2011. Chemoprevention by essential oil of turmeric leaves (Curcuma longa L.) on the growth of Aspergillus flavusand aflatoxin production. Food ChnemicalToxicol., 49: 1188-1192.

Škrinjar, M. M. and Nemet, N. T. (2009). Antimicrobial effects of spices and herbs essential oils. Acta Periodica Technologica, 40: 195-209.

Soković, M., Glamočlija, J., Marin, P.D., Brkić, D., van Griensven, J.L.D. 2010. Antibacterial Effects of the Essential Oils of Commonly Consumed Medicinal Herbs Using an In Vitro Model. Mol., 15: 7532-7546.

Souza, E.L., Stamford, T.L.M., Lima, E.O., Trajano, V.N., Filho, J.M.B. 2005. Antimicrobial effectiveness of spices: An approach for use in food conservation systems. Brazi. Arch. Biol. Technol., 48: 549-558.

Tajkarimi, M.M., Ibrahim, S.A., Cliver, D.O. 2010. Antimicrobial herb and spice compounds in food. Food Control, 21: 1199-1218.

Thanaboripat, D. 2011. Control of Aflatoxins in agricultural products using plant extracts. KMITL Sc. Tech. J., 11: 35-42.

Tripathi, P., Dubey, N.K., Shukla, A.K. 2008. Use of some essential oils as postharvest botanical fungicides in the management of grey mould of grapes caused by Botrytis cinerea. World J. Microbiol. Biotechnol., 24: 39-46.

Veldhuizen, E.J.A., Creutzberg, T.O., Burt, S.A., Haagsman, H.P. 2007. Low temperature and binding to food components inhibit the antibacterial activity of carvacrol against Listeria monocytogenes in steak tartare. J. Food Prote., 70: 2127-2132.

Yamazaki, K., Yamamoto, T., Yuji Kawai, Y., Inoue, N. 2004. Enhancement of antilisterial activity of essential oil constituents by nisin and diglycerol fatty acid ester. Food Microbiol., 21: 283289.

Yazdani, D., Ahmad, Z.A.M., How, T.Y., Jaganath, I.B., Shahnazi, S. 2013. Inhibition of aflatoxin biosynthesis in Aspergillus flavusby phenolic compounds extracted of Piper betleL. Iran. J. Microbiol., 5: 428-433.

\section{How to cite this article:}

Silviya R. Macwan, Bhumika K. Dabhi, K.D. Aparnathi and J.B. Prajapati. 2016. Essential Oils of Herbs and Spices: Their Antimicrobial Activity and Application in Preservation of Food. Int.J.Curr.Microbiol.App.Sci. 5(5): 885-901. doi: http://dx.doi.org/10.20546/ijcmas.2016.505.092 